\title{
OFICINAS EM MODALIDADE ONLINE: ESTUDANDO CONCEITOS DE CÁLCULO DIFERENCIAL E INTEGRAL UTILIZANDO O SYMPY LIVE
}

\author{
DOI: 10.37702/2175-957X.COBENGE.2021.3717
}

Lucas de Carvalho Barbosa - lucas.cbarbosa00@gmail.com

EE FURG

Rua das Algas 313

96215-500 - Rio Grande - RS

Bárbara Denicol do Amaral Rodriguez - barbararodriguez@furg.br IMEF FURG

Avenida Senador Tarso Dutra 2274

96216-010 - Rio Grande - RS

Cristiana Andrade Poffal - Poffal@gmail.com

IMEF FURG

Rua Mal Floriano Peixoto 256

96220-380 - Rio Grande - RS

Resumo: Este trabalho tem como objetivo apresentar novas formas de estudar os conceitos de Cálculo Diferencial e Integral e oportunizar a preparação do discente para a realidade atual e demanda do mercado de trabalho. Propõe-se a elaboração e o oferecimento de oficinas de aprendizagem utilizando a linguagem Python e suas potencialidades no estudo de conceitos acerca de limites, derivadas e integrais. Justifica- se a escolha da linguagem Python com o fato de ser gratuita, possuir uma sintaxe simples e intuitiva o que torna o aprendizado mais natural e fluido. As oficinas foram elaboradas para serem ofertadas presencialmente, mas devido à pandemia foram ministradas remotamente.

Palavras-chave: Cálculo. Python. Computação Simbólica. 


\section{OFICINAS EM MODALIDADE ONLINE: ESTUDANDO CONCEITOS DE CÁLCULO DIFERENCIAL E INTEGRAL UTILIZANDO O SYMPY LIVE}

\section{INTRODUÇÃO}

Nos últimos anos, houve um aumento considerável no número de alunos ingressantes no Ensino Superior. Para muitos, essa nova fase em suas vidas representa uma oportunidade de ascensão profissional ou a realização de um sonho. Mas, no decorrer do curso, muitos desistem pelos mais diversos motivos, sejam de ordem pessoal, contextual ou formativa. Esta realidade, por exemplo, é observada nos cursos de Engenharia da nossa Universidade, onde percebe-se, já nas disciplinais iniciais da grade curricular, dentre elas o Cálculo Diferencial e Integral, que grande parte destes "novos" discentes apresentam dificuldades na compreensão da notação e da linguagem e na construção de conceitos abstratos.

É neste cenário, e com o objetivo de diminuir os índices de evasão e retenção dos cursos, que o uso de recursos tecnológicos pode ser empregado como uma ferramenta útil na promoção de uma aprendizagem significativa e com um custo relativamente baixo. $A$ tecnologia nunca esteve tão evoluída como atualmente; com a globalização, estamos cada vez mais tendendo a uma espécie de "era digital", onde, os avanços tecnológicos se fazem presentes no cotidiano da grande maioria das pessoas (SILVA, 2019). Fernandes (2004, p. 43) complementa: "Hoje, com a velocidade de processamento e distribuição de informações via rede virtual, o computador tornou-se um instrumento indispensável para as realizações humanas." Para Flores et. al (2018), as tecnologias digitais fazem-se presentes nas mais distintas esferas da sociedade, seja na organização e na sistematização de informações, seja no comércio eletrônico, nas formas instantâneas de se comunicar e de se relacionar.

A Educação não ficou imune a essa "nova" forma de se relacionar. O uso da tecnologia possibilita uma "aprendizagem personalizada [...] ao estudante, pois permite ajustar-se melhor aos seus objetivos, ao seu estilo, à sua formação e ao seu ritmo de aprendizagem" (AMORIM et al. 2002, p. 8). A interação computador e indivíduo pode ser uma grande aliada no processo de ensino e aprendizagem. A partir dessa "nova" e favorável interação, o aluno será capaz de criar um ambiente propício para refletir, propor soluções e gerar novas ideias. Segundo Machado (2001), a dificuldade em ensinar Cálculo e matemática elementar nas Universidades, pode estar relacionada a uma imagem onde a matemática é o lugar das abstrações, seus aspectos formais não condizem com a realidade, tanto para quem aprende quanto para quem ensina. Neste caso, os softwares educacionais e as linguagens de programação são usados como um cenário, que possibilita ao aluno construir relações, ou pontes, entre os conceitos matemáticos e o mundo real. $A$ utilização de softwares educacionais e de linguagens de programação em laboratórios de informática vem se transformando em auxílios pedagógicos poderosos. Programar consiste em ordenar comandos para que o computador execute tais ordens através de um programa computacional, e com base numa linguagem de programação. Desenvolve, ainda, a capacidade de organizar as ideias e o pensamento estratégico para a solução de problemas.

Dentro deste contexto, objetivando apresentar novas formas de estudar os conceitos de Cálculo Diferencial e Integral e a preparação do discente para a realidade atual e demanda do mercado de trabalho, neste trabalho propõe-se a elaboração e o oferecimento de oficinas de aprendizagem utilizando a linguagem Python e suas potencialidades no estudo de conceitos acerca de limites, derivadas e integrais. A escolha da linguagem Python 
deve-se ao fato de que hoje ela vem sendo utilizada nos cursos da área tecnológica como a primeira disciplina de programação de computadores (Carvalhaes et al., 2019): é gratuita, possui uma sintaxe simples e intuitiva o que torna o aprendizado mais natural e fluido.

Para atingir os objetivos propostos, este trabalho está organizado da seguinte forma: a seção 2 apresenta um breve histórico do Python; a seção 3 relata a metodologia utilizada; a seção 4 contém os resultados e, por fim, a seção 5 , a conclusão e sugestões de continuidade dos estudos.

\section{BREVE HISTÓRICO DO PYTHON}

A Linguagem Python, é uma das mais conhecidas atualmente e foi projetada por Guido Van Rossum em 1989. Rossum, era um funcionário do CentrumWiskunde \& Informatica (Centro de Matemática e Ciência da Computação - CWI) em Amsterdã, na Holanda (Silva, 2019).

Rossum (1996) conta, que o nome "Python" vem de um grupo humorístico do cinema britânico Monty Python, criador do programa Monty Python Flying Circus. E também faz referência a cobra "píton". Segundo Leno (2014), no início de 1990 o Python já podia ser utilizado e, ao final do mesmo ano, a linguagem já havia superado a $A B C$ em termos de utilização no CWI. No ano seguinte, Guido foi remanejado da equipe Amoeba para a equipe Multimídia. E em 20 de fevereiro de 1991, foi publicada a primeira versão do Python, a v0.9.0. (também conhecida por Python 1.0), mas disponibilizada apenas em 1994. Leno (2014) afirma que em 2000, publicou-se a Python 2.0 e, posteriormente, em 6 de março de 2001, foi criada a Python Software Foundation (PSF), uma organização sem fins lucrativos, que se dedica à manutenção e desenvolvimento da linguagem de programação Python, composta por membros do grupo de desenvolvimento daquele período e por Eric Raymond (Silva, 2019). Já em 2008, para modernizar a linguagem, já que nem tudo era compatível com a versão anterior, surge o Python 3.0. Em 05 de outubro de 2020, foi lançado o Python 3.9. Leno (2014) ressalta que o Python está sob a licença Python Software Foundation License, que indica ser uma linguagem livre e de código aberto que permite modificações e estudos sobre o código.

Segundo Silva (2019), o Python possibilita ao usuário criar programas, sejam jogos ou códigos científicos para cálculos avançados, isso para vários ambientes, desde desktop, web ou até mesmo móvel, além de que, sua sintaxe é uma das suas mais simples dentre todas as linguagens de programação. Possui ampla biblioteca de suporte para programar junto com uma comunidade enorme, que pode auxiliar o usuário, através de diversos materiais em fóruns e redes sociais. O Python possui um amplo mercado, contando com grandes empresas usuárias de seus serviços. Atualmente é considerado uma linguagem de programação de alto nível e é muito utilizada em diversos meios (Carvalhaes et al., 2019). Desta forma, inicialmente, para o desenvolvimento das oficinas, foi utilizado o "Integrated Development and Learning Environment" (IDLE), um ambiente de desenvolvimento para o Python. Neste ambiente é possível utilizar o Python em conjunto com suas bibliotecas. Para a elaboração das primeiras oficinas foram utilizadas as bibliotecas SymPy (versão 1.5.1) e Matplotlib (versão 3.2.2).

O SymPy é uma vasta biblioteca que possui ferramentas de álgebra computacional, é nele que encontramos os recursos para estudar conteúdos como limites, derivadas e integrais. Essa biblioteca está disponível, ainda, de forma online nos sítios de internet: SymPyLive (https://live.sympy.org/) e SymPy Gamma (https://gamma.sympy.org/) E por fim, para trabalhar com gráficos utiliza-se o Matplotlib, o qual fornece vários recursos para a plotagem. 


\section{METODOLOGIA}

Inicialmente foi elaborado um tutorial de instalação do Python e suas bibliotecas, com o objetivo de auxiliar os alunos a se familiarizarem com a linguagem e parte de seus recursos. Nele é realizada a demonstração de cada passo necessário para realizar a instalação e uma breve introdução ao IDLE. Neste ambiente é possível fazer do Python uma simples calculadora, utilizar comandos para trabalhar a matemática básica. Entretanto, para o estudo de conteúdos de Cálculo Diferencial e Integral são necessários recursos que não estão disponibilizados no IDLE, a partir disso o emprego da computação simbólica torna-se necessário. Nela, símbolos literais, que não possuem um valor atribuído, são trabalhados, o que nos permite utilizá-los como variáveis.

Já com o tutorial pronto, o próximo passo foi dar início à elaboração das oficinas de aprendizagem. Cada oficina elaborada aborda um tema específico, contendo ilustrações, exemplos e questões para exercitar cada tópico apresentado. Uma questão central (Figura 1) foi escolhida para nortear os trabalhos e simulações. Sua solução, bem como os comandos do Python utilizados são apresentados ao final de cada oficina.

Figura 1- Questão Norteadora da oficina sobre limites
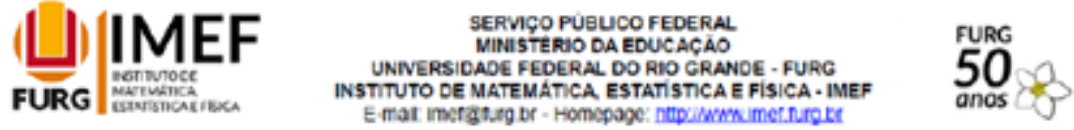

Para finalizarmos nossa oficina retomamos à Questão Norteadora: Durante uma epidemia de dengue, o número de pessoas que adoeceram, num certo bairro, após t dias é dado por:

$L(t)=\frac{100000}{1+19900 e^{-0.8 t}}$

Determine a quantidade máxima de individuos atingidos pela epidemia. Vamos resolver este problema utilizando os comandos do Python. Para isso devemos:

1) Utilizar o comando def para definir nossa função $L(t)$;

2) Calcular o limite de $\mathrm{L}(\mathrm{t})$ a fim de determinar a quantidade máxima de individuos atingidos pela epidemia.

Veja a solução na Figura 5.

Figura 5: Solução da questão norteadora.

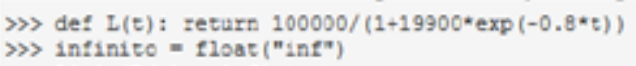

》 1 init (L ( $t), t, i n f i n i t o)$

100000

Fonte: Próprio autor.

Na primeira oficina intitulada, "A Computação Simbólica e o Python" (Figura 2), é apresentada aos alunos uma introdução ao Python e suas bibliotecas, utilizando o IDLE como ferramenta de estudo. Essa oficina fornece a base de comandos necessários para desenvolver as demais. Os tópicos são apresentados de forma simples, por meio de exemplos e ilustrações e abordam: importação de bibliotecas, declaração de variáveis como símbolos, atribuição de valores, comandos para simplificação, declaração de funções matemáticas, resolução de equações e inequações, entre outros. 
Figura 2 - Oficina sobre comandos e operações básicas.
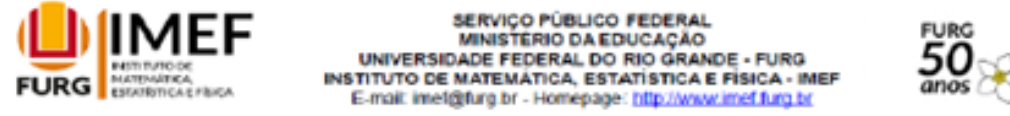

Oficina 1 - A Computação Simbólica e o Python

Objetivo: Fornecer ao aluno conhecimentos básicos para o desenvolvimento dos conceitos do Cálculo Diferencial e Integral utilizando a linguagem de programação Python.

Duração: 2 horas/aula.

Requisitos: Ter instalado o Python (Versão 3.8.1 64bits), SymPy (Versão 1.0 32bits) e Numpy (Versão 1.7.1 64bits)

Questão norteadora:

1. Comandos e Operaçōes Básicas

Atribuição de valores: no Pyhton para fazer a atribuição de um valor a uma variável basta digitar a variável, o sinal de igual " $=$ ", seguido do valor que desejamos atribuir. Observe o exemplo na Figura 1.

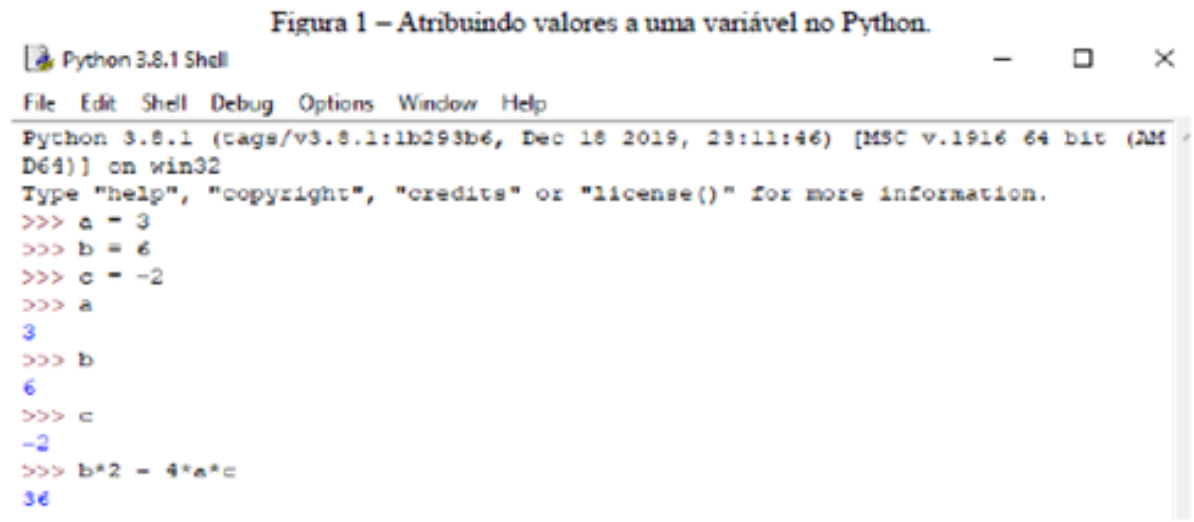

Fonte: Próprio autor.

Após a primeira oficina, com base no material didático (Rodriguez, Meneghetti, Poffal, 2016a, 2016b, 2016c), foi desenvolvido o material para as novas oficinas de aprendizagem, abordando temas específicos de Cálculo Diferencial e Integral, tais como: limites, derivadas e integrais.

A fim de tornar as oficinas mais acessíveis aos alunos, evitando o "desperdício" do tempo com o processo de instalação do IDLE, que para muitos pode ser um processo longo e confuso, optou-se por adaptá-las ao SymPy Live (https://live.sympy.org/), uma plataforma online na qual está disponível a biblioteca SymPy (Figura 3). Sua disponibilidade online facilita a aplicação das oficinas e aumenta a acessibilidade à utilização do Python, pois permite trabalhar até mesmo em um smartphone, descartando a necessidade de possuir um notebook ou computador ou estar em um laboratório de informática: é preciso apenas do aparelho e conexão com a internet.

Inicialmente, as oficinas seriam oferecidas na forma presencial, mas devido à pandemia do COVID-19, adaptações foram necessárias: oferecimento na modalidade online e a gravação na forma de vídeo apresentando os conteúdos abordados. Para cada oferta foi elaborado um convite (Figura 4) e este divulgado no site da Universidade, bem como nas redes sociais. Para a aplicação das oficinas, foram desenvolvidos formulários de inscrição e as vagas eram limitadas. 
Figura 3 - O SymPy Live.

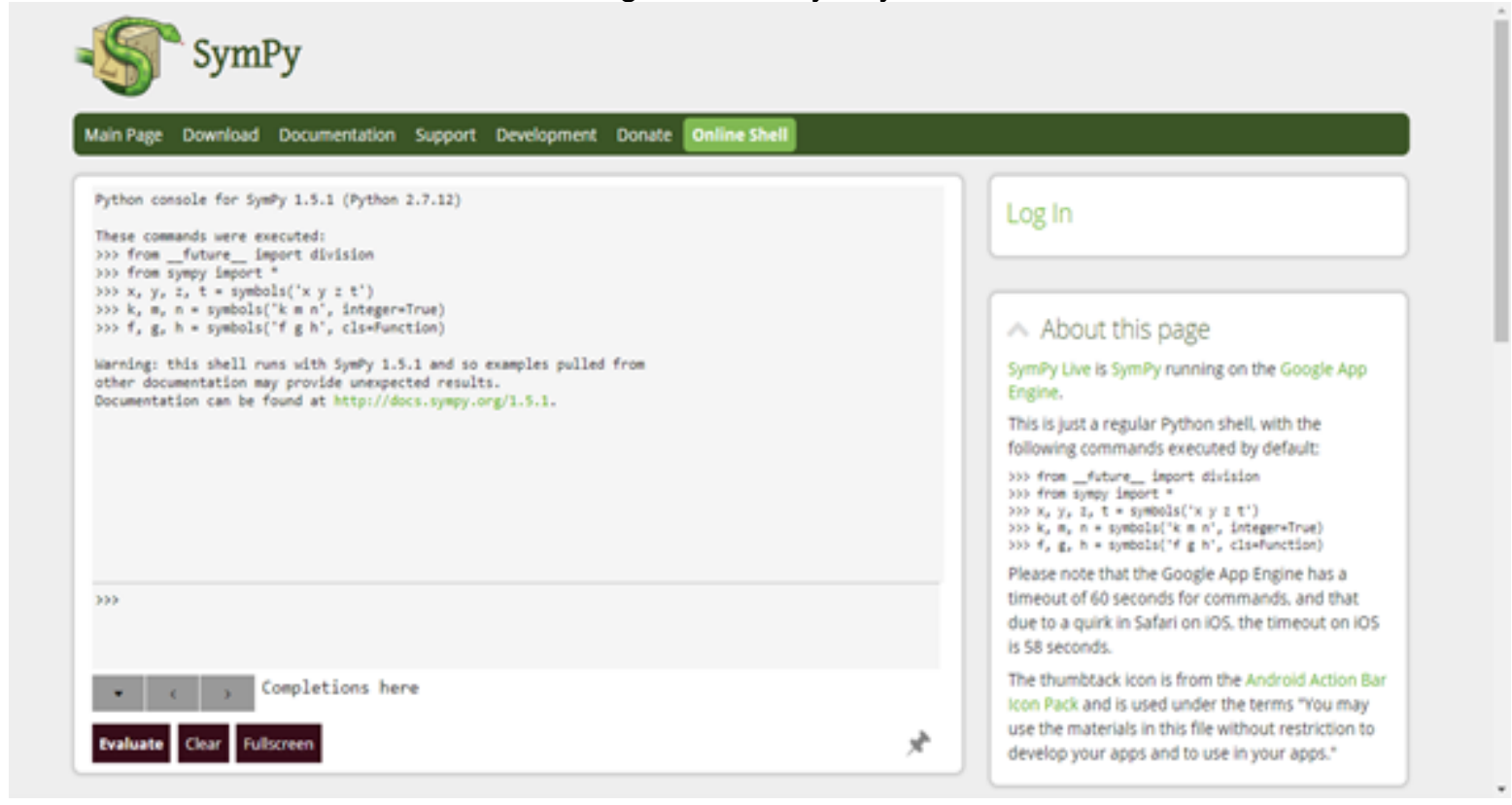

Fonte: do autor.

Figura 4 - Convite

\section{题IIMEF}

O projeto "O Ensino de Cálculo Diferencial e Integral utilizando a linguagem Python" convida você a participar da oficina online

Limites de funções com o Python

Data: 29 de setembro, $19 \mathrm{~h}$ às $27 \mathrm{~h}$

Local: Google Meet

Inscriçôes: https://forms.gle/ whkaJPsuyDkjghvL6

Vagas limitadas!

A sala virtual será enviada por e-mail aos participantes inscritos

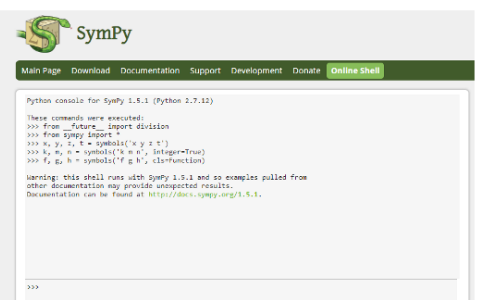

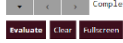

Ação do Grupo de Pesquisa LEMAS - https://lemas.furg.br/

Fonte: do autor.

\section{RESULTADOS}

Até o presente momento, foram elaborados um tutorial de instalação e oito oficinas abordando: comandos e operações básicas do Python, limites, continuidade, derivadas, derivadas parciais, integrais indefinidas e os métodos de integração, integrais definidas, integrais múltiplas, aplicações de integrais (cálculo de área, cálculo do comprimento do arco, volume de sólidos de revolução). 


\section{Evento Online}

"Formação em Engenharia: Tecnologia, Inovação e Sustentabilidade"

Foram ministradas três oficinas com uma reoferta na modalidade online. A primeira oficina intitulada "Limites de funções com o Python" foi realizada nos dias 29 de setembro e 22 de outubro (Figura 5). Para um melhor aproveitamento da oficina por parte dos alunos, uma adaptação foi necessária, a fim de apresentar comandos básicos antes de abordar os conceitos de limites.

Figura 5 - Oficina "Limites de funções com o Python"
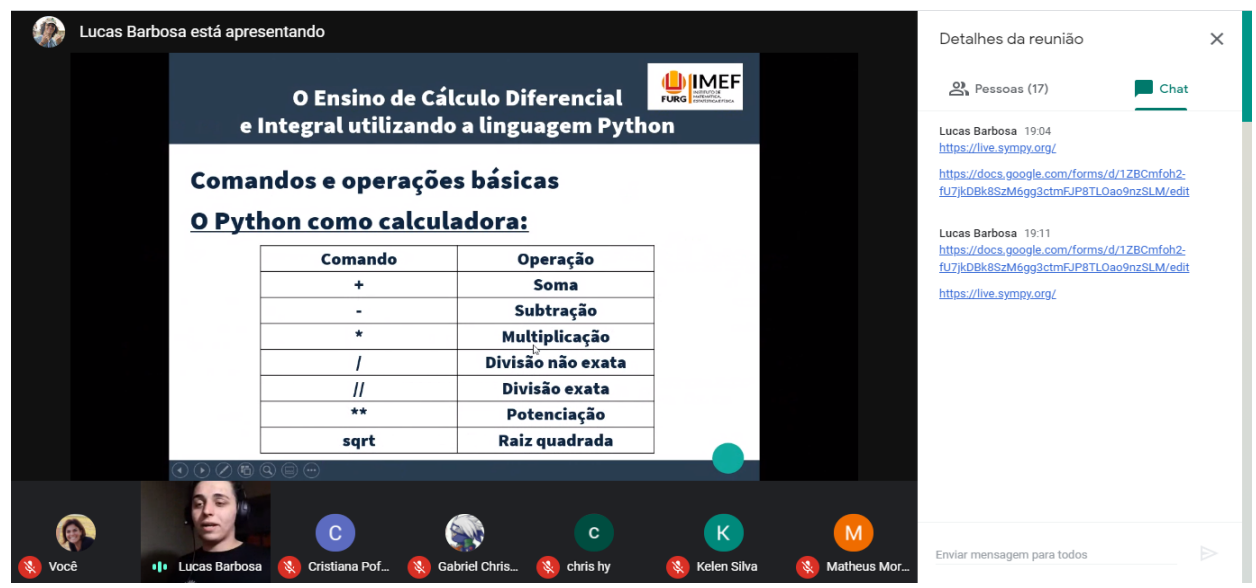

Fonte: do autor.

A segunda oficina intitulada "Derivadas de funções com o Python" aconteceu no dia 29 de outubro (Figura 6). Já a terceira, "Integrais e aplicações com o Python", no dia 16 de novembro. Todas as oficinas foram realizadas às $19 \mathrm{~h}$ no Google Meet ao longo do ano de 2020.

Figura 6 - Oficina "Derivadas de funções com o Python".

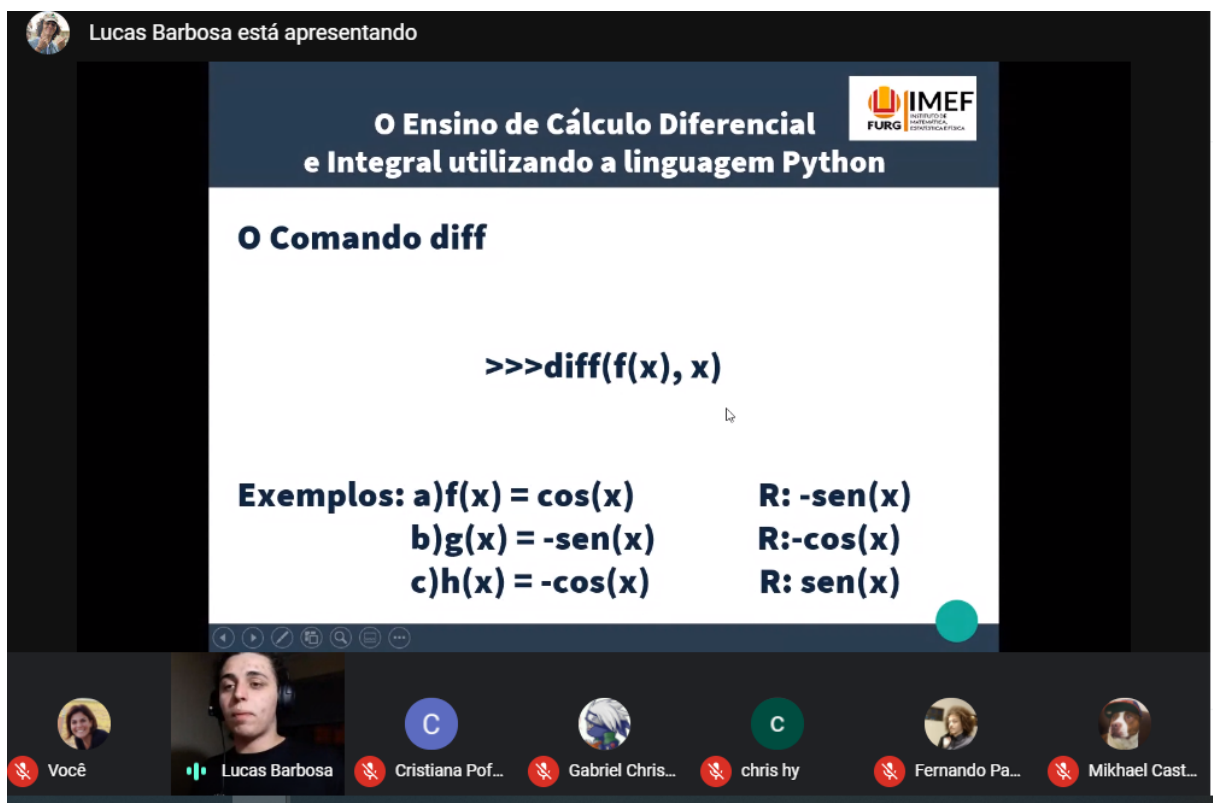

Fonte: do autor. 
Ao final de cada oficina foi solicitado aos participantes o preenchimento de um formulário de avaliação. Os dados encontram-se em fase de análise.

Para dar continuidade à elaboração e oferecimento de novas oficinas, por meio de um formulário do Google, realizou-se uma consulta aos docentes do Instituto de Matemática, Física e Estatística (IMEF), a fim de investigar quais eram as principais dúvidas de seus alunos acerca dos conteúdos de Cálculo e quais seriam os temas de maior interesse para futuras oficinas. Para os discentes, foi desenvolvido um formulário a fim de investigar suas dificuldades e seu interesse em participar de alguma das oficinas do projeto. O levantamento destes dados está em fase de execução.

\section{CONSIDERAÇÕES FINAIS}

Os recursos tecnológicos podem fornecer infinitas possibilidades para a compreensão e visualização de conceitos, facilitando o entendimento de notações e linguagens. Entretanto, para uma nova configuração das práticas educativas já existentes são necessárias ações que "coloquem" o estudante como um sujeito ativo no processo de aprendizagem. A criação de situações nas quais os discentes possam interagir, trocar ideias e argumentar, trará significado aos conceitos e permitirá aos acadêmicos a aprendizagem de novas habilidades. Acredita-se que com o oferecimento das oficinas aos estudantes foi possível instigá-los a refletir, auxiliá-los na construção do conhecimento matemático de uma maneira mais significativa e contribuir, desta forma, para uma diminuição dos índices de retenção e evasão em disciplinas de Matemática. Mas acima de tudo, para a formação de acadêmicos melhor capacitados para atuar no mercado profissional. A flexibilização para o formato online permitiu, ainda, estender as dimensões de tempo e de espaço fornecidas pelas tecnologias digitais.

Além disso, a realização das oficinas permitiu promover ações a fim de estimular a construção de uma cultura de uso de softwares livres como uma forma de valorizar a busca pelo conhecimento. Bem como, empregar uma linguagem de programação simples, mas poderosa, e que vem sendo utilizada por inúmeros profissionais nos mais diversos campos da Ciência. A partir da elaboração das oficinas foi possível revisitar conceitos matemáticos estudados nas disciplinas iniciais de matemática dos cursos de Engenharia para aplicá-los na resolução de problemas através da utilização da linguagem de programação Python.

Muito ainda precisa-se avançar, principalmente no sentido de fomentar ações que preponderam a criatividade, o senso crítico e a interação, mas as oficinas neste trabalho apresentadas representam uma forma alternativa de ensino e aprendizagem e buscam contribuir para que essa realidade mude e para alcançar resultados positivos em relação ao número de aprovações e à redução da evasão em disciplinas de Cálculo.

\section{REFERÊNCIAS}

CARVALHAES, Claudio Gonçalves, et al. Cálculo Diferencial e Integral de Funções de uma Variável com Python. 1. ed. Rio de Janeiro: Ciência Moderna, 2019.

FERNANDES, Natal L. R. Professores e computadores: Navegar é preciso. Porto Alegre: Mediação, 2004. 
FLORES, Jeronimo Becker, LIMA, Valderez Marina do Rosário Lima, MÜLLER, Thaísa JACINTHO. O uso das Tecnologias da Informação e Comunicação no ensino de Cálculo Diferencial e Integral: reflexões a partir de uma metanálise. Abakos, Belo Horizonte, v. 6, n. 2, p. 21-35, 2018.

LENO, Magnun. A História do Python. 2014. Disponível em: http://mindbending.org/pt/ahistoria-do-python. Acesso em 13 de abr. de 2021.

RODRIGUEZ, Bárbara, MENEGHETTI, Cinthya, POFFAL, Cristiana. Continuidade de funções de uma variável real. Rio Grande: FURG, 2016a.

Derivadas de funções de uma variável real. Rio Grande: FURG, 2016b.

Limites de funções de uma variável real. Rio Grande: FURG, 2016c.

ROSSUM, Guido Van. Foreword for "Programming Python. Reston, VA, May 1996.

SILVA, João Elder Laurentino da. Comparando as Linguagens de Programação Fortran e Python através de Problemas Matemáticos. Trabalho de Conclusão do Curso de Licenciatura em Matemática, da Universidade Federal de Campina Grande Campus Cuité (PB), 2019. Disponível em:

http://dspace.sti.ufcg.edu.br:8080/jspui/bitstream/riufcg/8382/1/JO\%C3\%830\%20ELDER \%20LAURENTINO\%20DA\%20SILVA\%20-

\%20\%20TCC\%20\%20MATEM\%C3\%81TICA\%20\%202019.pdf. Acesso em:09 mai.2021.

\title{
ONLINE WORKSHOPS: STUDYING DIFFERENTIAL AND INTEGRAL CALCULUS CONCEPTS USING SYMPY LIVE
}

\begin{abstract}
This work aims to present new ways of studying the concepts of Differential and Integral Calculus and provide opportunities for the preparation of students for the current reality and demand of the labor market. It is proposed the elaboration and offer of learning workshops using the Python language and its potentialities in the study of concepts about limits, derivatives and integrals. The choice of the Python language is justified by the fact that it is free, has a simple and intuitive syntax which makes learning more natural and fluid. The workshops were designed to be offered in person, but due to the pandemic they were given remotely.
\end{abstract}

Keywords: Calculus. Python. Symbolic computing. 